Revue des patrimoines

\title{
Chronologie pariétale des œuvres magdaléniennes du Roc-aux-Sorciers (Angles-sur-l'Anglin, Vienne) : entre tradition et innovation
}

\section{Geneviève Pinçon}

\section{OpenEdition}

Journals

Édition électronique

URL : http://journals.openedition.org/insitu/3292

DOI : $10.4000 /$ insitu.3292

ISSN : 1630-7305

Éditeur

Ministère de la culture

Référence électronique

Geneviève Pinçon, «Chronologie pariétale des œuvres magdaléniennes du Roc-aux-Sorciers (Anglessur-l'Anglin, Vienne) : entre tradition et innovation », In Situ [En ligne], 9 | 2008, mis en ligne le 18 avril 2012, consulté le 01 mai 2019. URL : http://journals.openedition.org/insitu/3292 ; DOI : 10.4000/ insitu.3292

Ce document a été généré automatiquement le 1 mai 2019.

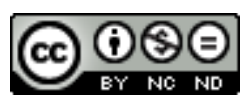

In Situ Revues des patrimoines est mis à disposition selon les termes de la licence Creative Commons Attribution - Pas d'Utilisation Commerciale - Pas de Modification 4.0 International. 


\title{
Chronologie pariétale des œuvres magdaléniennes du Roc-aux- Sorciers (Angles-sur-l'Anglin, Vienne) : entre tradition et innovation
}

\author{
Geneviève Pinçon
}

\section{Introduction}

1 Dater une œuvre paléolithique, la rattacher à une culture est une première étape. On sait combien elle peut être difficile, notamment pour les œuvres réalisées sur les parois des grottes profondes comme Lascaux ou Chauvet où les traces de séjour sont rares, malgré l'apport des techniques de datations directes de plus en plus précises.

2 Il existe plusieurs façons de dater une œuvre pariétale paléolithique. La plus courante est la datation relative. Elle peut être réalisée par attribution stylistique, caractérisée aussi bien par les techniques d'exécution, les couleurs utilisées, les espèces représentées et les formes de représentations de leurs corpulences ou de leurs détails anatomiques, les signes associés, etc. Cette méthode a été largement employée lors de la détermination des premières chronologies de l'art pariétal (Breuil ${ }^{1}$, Leroi-Gourhan ${ }^{2}$ ). Elle le demeure encore à défaut de tout repère chronologique absolu et constitue une véritable problématique dans l'analyse des œuvres d'art pariétal paléolithique. Cependant, les nouvelles découvertes comme la grotte Chauvet témoignent de la fragilité de ces comparaisons stylistiques et de leur attribution chronologique.

3 Les abris sous-roches sculptés, dont la particularité est d'associer in situ les œuvres pariétales et les niveaux d'occupation, permettent une datation relative des œuvres, du fait de leur recouvrement par les sédiments et la mise au jour de fragments d'œuvre pariétale au sein même des couches archéologiques dont la caractérisation peut être 
établie sur différents critères (attribution culturelle, datation relative, datation absolue sur certains matériels archéologiques).

4 Les conditions de découverte du Roc-aux-Sorciers à Angles-sur-l'Anglin dans le département de la Vienne, permettent d'évoquer la chronologie des œuvres pariétales monumentales qu'il renferme. En s'appuyant sur un relevé analytique, indispensable pour une bonne compréhension de l'art pariétal, nous constatons des phénomènes de retaille et des vestiges de sculpture volontairement effacés. Ceux-ci apportent des précisions quant à la chronologie des œuvres pariétales. La «lecture archéologique» de la paroi amène à une "chronologie pariétale » traduisant l'évolution de la paroi ornée, le changement des thèmes artistiques et des choix iconographiques au cours de l'occupation magdalénienne de ce site.

\section{Le contexte archéologique des œuvres pariétales du Roc-aux-Sorciers}

Parce qu'il fut découvert dans son contexte archéologique, le Roc-aux-Sorciers constitue un site d'art pariétal exceptionnel. Cette conjoncture permet de bien dater les œuvres pariétales, au-delà d'une approche stylistique quelquefois trop aléatoire, et d'aborder le comportement des hommes du Paléolithique supérieur. L'art pariétal associé à un sol d'occupation offre les meilleures conditions d'appréhension de la place de leur système symbolique dans leur vie quotidienne. Quel était, il y a 15000 ans, le rôle de cet art associé à la découverte de vestiges d'occupation magdalénienne? Quel rapport entretenaient les Magdaléniens avec cet art? Etait-ce un art lié au vécu quotidien, ou plutôt à des actes exceptionnels? Cependant, «ni le critère d'emplacement ni l'association ou l'isolement par rapport aux habitats ne suffisent à caractériser un art sacré ni à écarter l'idée du sacré » (Lorblanchet ${ }^{3}, 2002$, p. 104). 
Figure 1

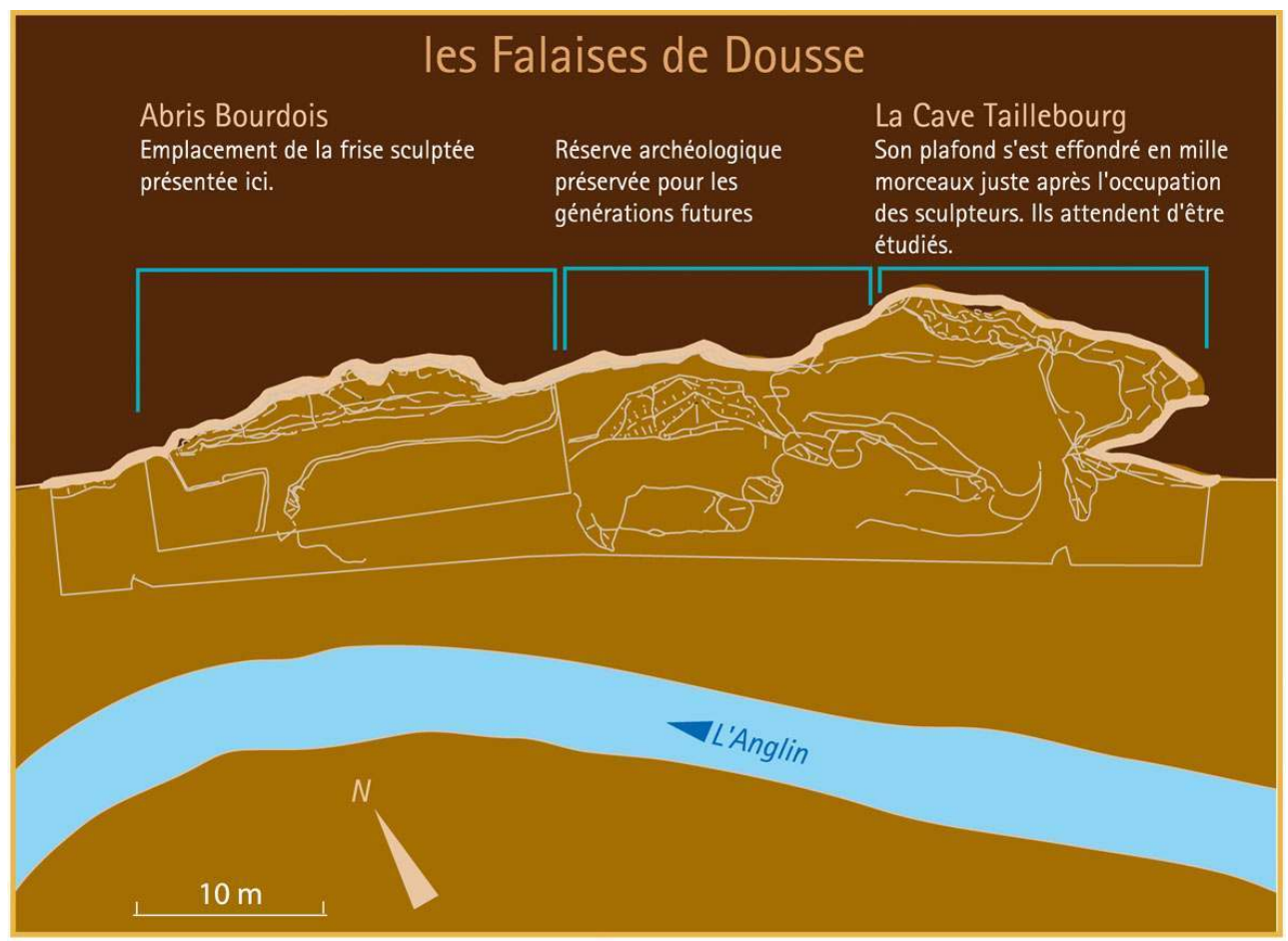

Plan général du site du Roc-aux-Sorciers

(c) G. Pinçon, Gulliver d'après le relevé topographique de F. Rouzaud, Y. Le Guillou. (Pinçon, Geneviève. Rapport d'activité sur le site du Roc-aux-Sorciers à Angles-sur-l'Anglin (Vienne), 1993)

Exposé plein sud, au pied des falaises jurassiques de Dousse, sur la rive droite de l'Anglin, ce gisement, classé Monument historique le 18 janvier $1955^{4}$, s'étend sur une cinquantaine de mètres (fig. $\mathbf{n}^{\circ} \mathbf{1}$ ). Dans cet habitat en pied de falaise, découvert en 1927, Lucien Rousseau ${ }^{5}$ identifie la culture du Magdalénien moyen. Il fouille un remplissage issu d'éboulements successifs sans y repérer de blocs sculptés. Ce sont deux grandes dames de la Préhistoire qui mettent au jour l'art pariétal dans son contexte archéologique. Suzanne de Saint-Mathurin ${ }^{6}$ et Dorothy Garrod ${ }^{7}$ commencent les recherches à proximité de celles de leur prédécesseur, dans la Cave Taillebourg. Elles fouillent le gisement de 1947 à 1964 et recueillent plus de 350 blocs sculptés, gravés et peints ${ }^{8}$. Ces blocs proviennent du plafond de l'abri ; elles en ont la certitude après avoir découvert un bison sculpté encore in situ (fig. $\mathbf{n}^{\circ}$ 2). 
Figure 2

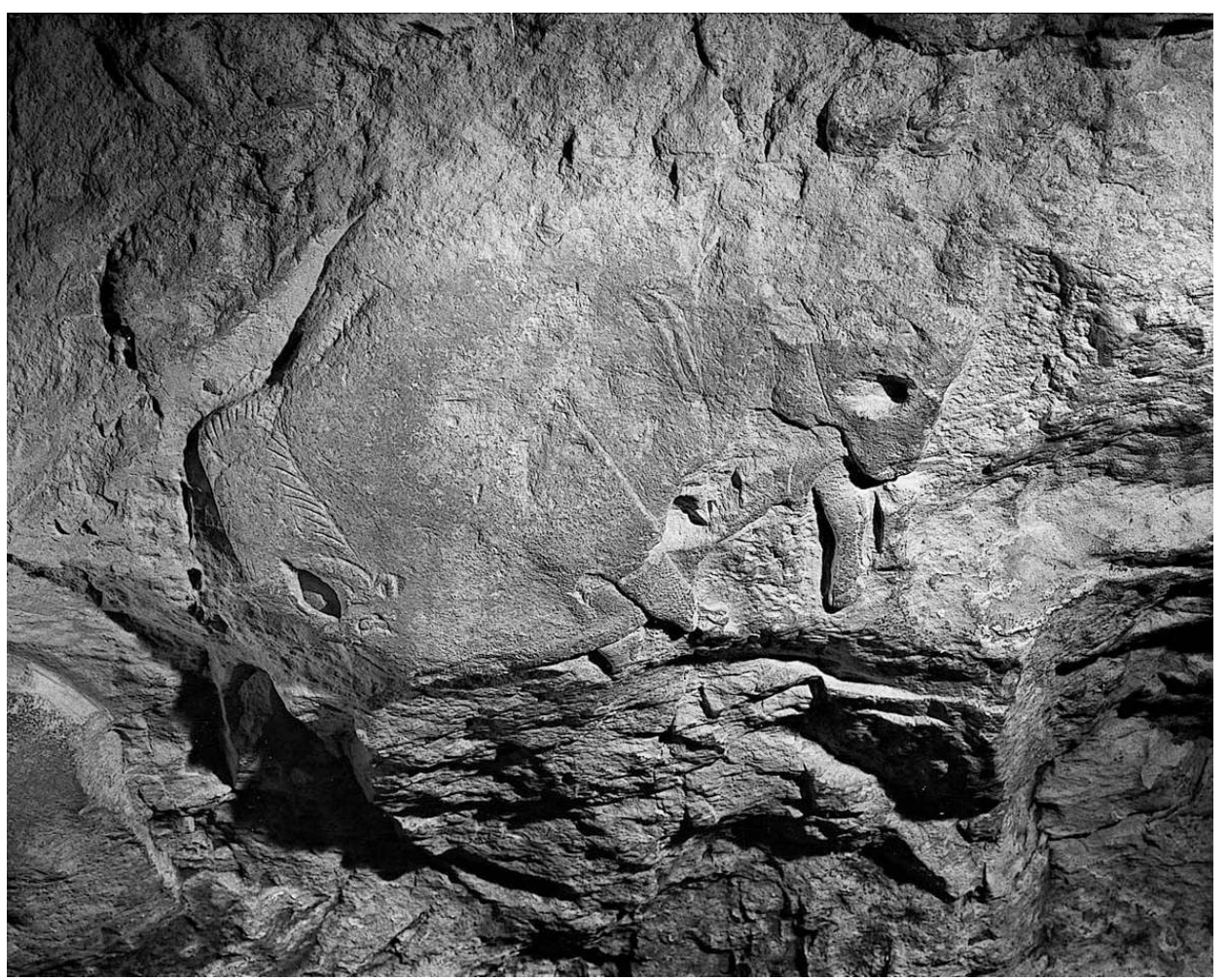

Bison sculpté encore in situ, plafond de la cave Taillebourg. de Saint-Mathurin, S

(c) Musée d'archéologie nationale, fonds Saint-Mathurin

En effet, cette partie de l'abri a été fortement perturbée par un effondrement qui a entrainé une fouille complexe mettant en évidence une cavité relativement importante dans laquelle l'homme magdalénien s'est installé.

En 1950, quelques 30 mètres plus en aval, elles dégagent la paroi et butent rapidement sur un cheval sculpté en fond d'abri, en position "agnus dei". C'est le commencement du dégagement de la frise sculptée magdalénienne de l'abri Bourdois, encore en place sur plus de 18 mètres de long (fig. $n^{\circ}$ 3). L'abri Bourdois a moins souffert de cette période d'effondrement. Il est un témoin assez unique offrant encore aujourd'hui l'art pariétal dans son contexte archéologique. Le remplissage sédimentaire de l'Abri Bourdois, qui recouvrait l'ensemble de la frise sculptée encore in situ, présente une stratigraphie qui se partage en deux ensembles principaux, le Magdalénien moyen et le Magdalénien supérieur (fig. $n^{\circ}$ 4). Les Magdaléniens se sont installés directement sur le socle rocheux, le long de la paroi, marquant leur présence par des foyers importants associés à beaucoup de matériel typique. Plusieurs blocs sculptés y ont été retrouvés attestant de leur contemporanéité avec les premières occupations du Magdalénien moyen. 
Figure 3

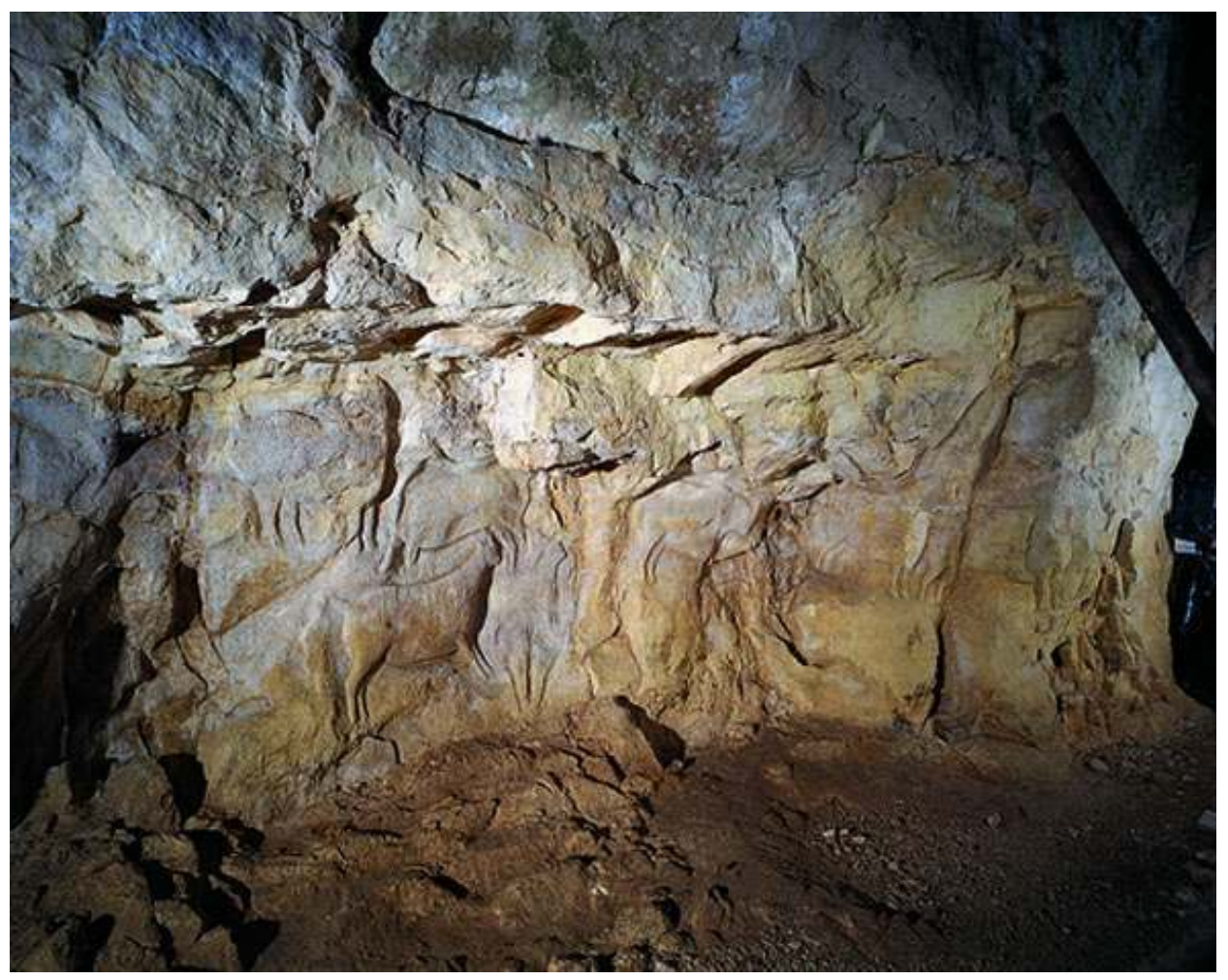

Frise sculptée magdalénienne de l'abri Bourdois, encore en place sur plus de 18 mètres de long Maulny, A. (C) G. Pinçon et DRAC Poitou-Charentes, 1997 
Figure 4

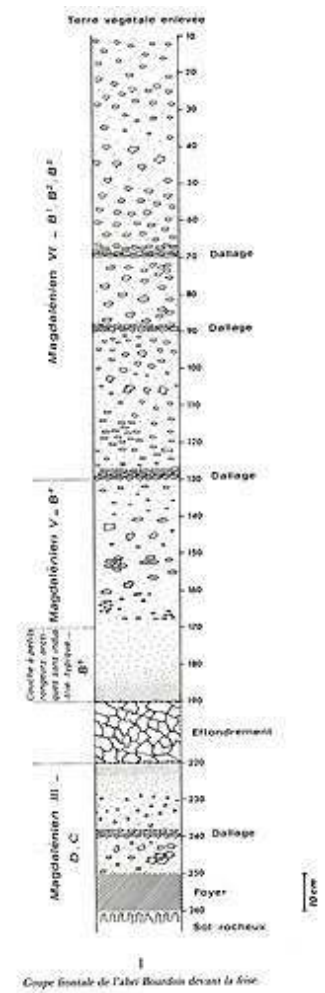

Relevé schématique de la stratigraphie de l'abri Bourdois

(c) S. de Saint-Mathurin (D'après Saint-Mathurin, Suzanne. L'Abri du Roc-aux-Sorciers. L'Art des

Cavernes. Atlas des grottes ornées paléolithiques françaises. Paris : Ministère de la Culture, Impr. Nat., 1984. p. 283-287)

\section{Description de la stratigraphie dans l'abri Bourdois proposée par Suzanne de Saint-Mathurin}

- ensemble du Magdalénien supérieur où se discernent plusieurs occupations sporadiques de chasseurs cueilleurs magdaléniens

[Couche RSB2 : $12570 \pm 140$ ans B.P. (GifA 94189)].

[Couches RSB2 + RSB3 : 11.565 \pm 130 ans B.P. (GRN 2916)(d'après archives S. de SaintMathurin)]

couche B3, B2, B1 : couche argilo-sableuse séparée par des dallages discontinus composés de galets de rivières, industrie du Magdalénien supérieur

[Couche RSB4 : 10.800 \pm 120 ans B.P. (GRN 2912) (d'après archives S. de Saint-Mathurin)]

[Couche RSB4 : 13.720 \pm 140 ans B.P. (GifA 94192)],

couche B5 : couche sableuse faisant suite à l'effondrement avec peu d'industrie

couche B4 : couche de limon lœssoïde avec industrie du Magdalénien supérieur

10 - ensemble du Magdalénien moyen où se distinguent différentes couches d'occupation des sculpteurs magdaléniens

effondrement

Cet éboulement a scellé les couches du Magdalénien moyen. Celui-ci fut mineur dans l'abri Bourdois.

[Couche RSC1 : $14.770 \pm 140$ ans B.P. (GifA 94190)] 
couche $\mathrm{C}$ : couche argilo sableuse de couleur jaune clair

dallage en pierres calcaires et galets de rivière

[Couche RDC3 =RSD : $14.510 \pm 160$ ans B.P. (GifA 94191)]

[Couche RSD : $14.160 \pm 80$ ans B.P. (GrN 1913) (Saint-Mathurin, 1984, p. 586)]

couche $\mathrm{D}$ : industrie abondante du Magdalénien moyen, foyers

couche $\mathrm{E}$ : couche fortement ocrée

couche $\mathrm{F}$ : sable jaunâtre

[14.030 \pm 100 ans B.P. (GRO 1913) (d'après archives S. de Saint-Mathurin)]

11 Lorsque les magdaléniens ont commencé à occuper le site, les configurations morphologiques des deux parties, aval et amont, du gisement étaient bien différentes, et offraient des possibilités d'aménagement variées. La description qu'en donnent J. Lorenz et J.-P. Gély, géologues, est la suivante ${ }^{9}$ :

12 «La Cave Taillebourg est une large cavité karstique en pied de falaise. La grotte se développe parallèlement à la falaise et se termine, à l'est, par des conduits karstiques très étroits, développés sur des fractures tectoniques d'orientation N100. Les calcaires bioclastiques, à la base de la cavité, sont gélifractés. Le long de la paroi, la masse gélifractée est fracturée et déstructurée. Elle représente un important volume de roche décomprimée par effet de versant, sur une profondeur de l'ordre de 2 ou 3 mètres. En revanche, la partie haute de la grotte creusée dans les calcaires à polypiers, massifs et résistants, n'est pas altérée par la gélifraction. Ainsi, la voûte de la cave Taillebourg est saine mais peut avoir un aspect très irrégulier du fait de la présence des amas de polypiers noyés dans les calcaires massifs. La cavité karstique bien développée forme un lieu abrité et facile à fermer, probablement par une cloison. Seul son plafond offre une paroi saine, pouvant supporter les œuvres gravées ou sculptées tandis que la paroi verticale en fond d'abri de très mauvaise qualité, en est inapte. La datation de ces œuvres pariétales en plafond aurait été plus difficile si elles étaient encore in situ. La découverte des blocs ornés issus du plafond positionnés dans la stratigraphie permet de les attribuer sans ambiguïté au Magdalénien moyen.

13 L'abri Bourdois est un abri de pied de falaise, peu développé. A la base de la cavité, comme dans la cave Taillebourg, une masse gélifractée de calcaires bioclastiques devait exister. La purge de pied de paroi a eu lieu probablement à la suite d'un effondrement antémagdalénien de pan de falaise, donnant accès à une paroi assez saine en fond d'abri. La partie supérieure de l'abri, présente une surface karstifiée de calcaires à polypiers comme dans la Cave Taillebourg. L'abri Bourdois est très ouvert et peu prononcé, avec quelques mètres d'avancée de falaise tout au plus, où l'état de surface du fond de l'abri est convenable pour les gravures et les sculptures.»

14 Ainsi, le fond d'abri en aval et le plafond en amont sont les supports les plus aptes à recevoir les œuvres pariétales au moment où les magdaléniens occupent le site du Rocaux-Sorciers. Leur mise au jour dans un contexte archéologique précis permet déjà de dater ces œuvres du Magdalénien moyen, il y a 14000-15000 ans.

\section{La frise sculptée du Roc-aux-Sorciers ${ }^{10}$}


Figure 5

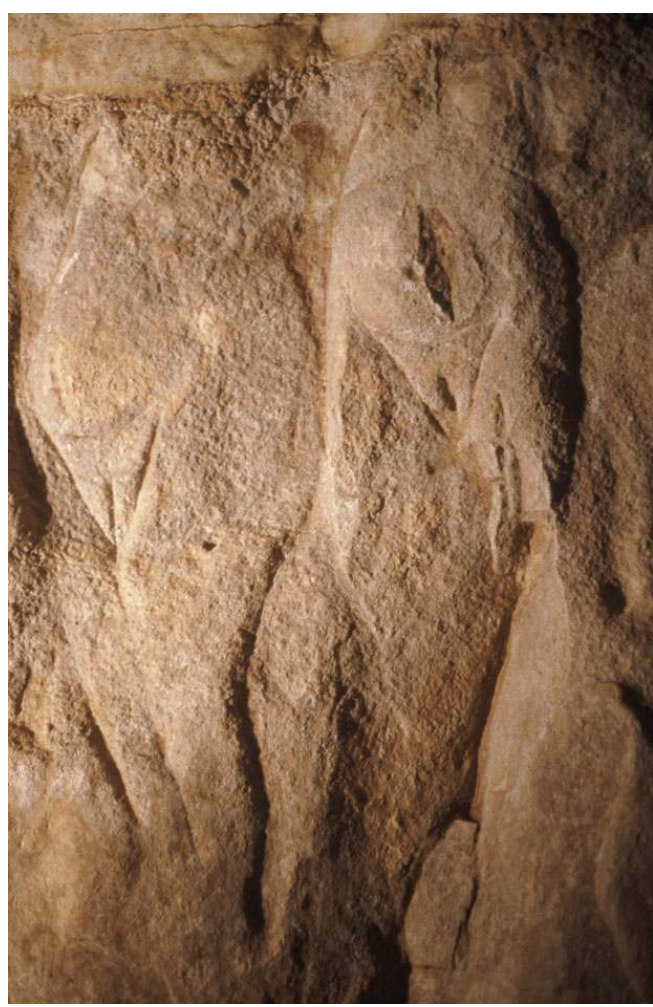

Gravures fines

Pinçon, G. (C) G. Pinçon et DRAC Poitou-Charentes, 1994

Sur la frise en place de l'abri Bourdois, se côtoient, de façon très organisée, des bisons, chevaux, femmes (fig. $\mathbf{n}^{\circ}$ ), félins, bouquetins,... et autres thèmes dont plusieurs sont représentés grandeur nature. Les techniques allient hauts-reliefs, bas-reliefs, gravures et peintures. De nombreux anneaux, ayant pu être utilisés, participent à la rythmique de l'organisation des figures. L'abondance d'animaux figurés, leur mise en scène, ainsi que la forte présence du thème humain sont des éléments essentiels de cet art. Plusieurs tonalités, rouge vif, rouge-brun et jaune, comme sur le bloc portant une tête d'homme sculptée, gravée et peinte, ont été relevées. La couleur rouge se retrouve également, sous forme de ponctuations disposées en bandes sur les sujets sculptés ou gravés.

Cet art naturaliste monumental, présentant des sujets aux proportions respectées, aux détails précis et aux attitudes animées, offre également des éléments de style comme la géométrisation de détails anatomiques, des attitudes répétées comme l'inclinaison de la tête des chevaux vers leurs pattes antérieures ou la position "pattes repliées » d'autres sujets. Le positionnement des figures sur des éléments naturels de la paroi est également récurrent, comme les bisons dont la tête s'appuie systématiquement sur une arête naturelle. Des procédés techniques renforcent l'homogénéité des figures, comme la légère torsion appliquée aux mufles des chevaux ou des bouquetins mettant en valeur les deux naseaux vus de face, alors que le reste de la tête est représenté de profil. 


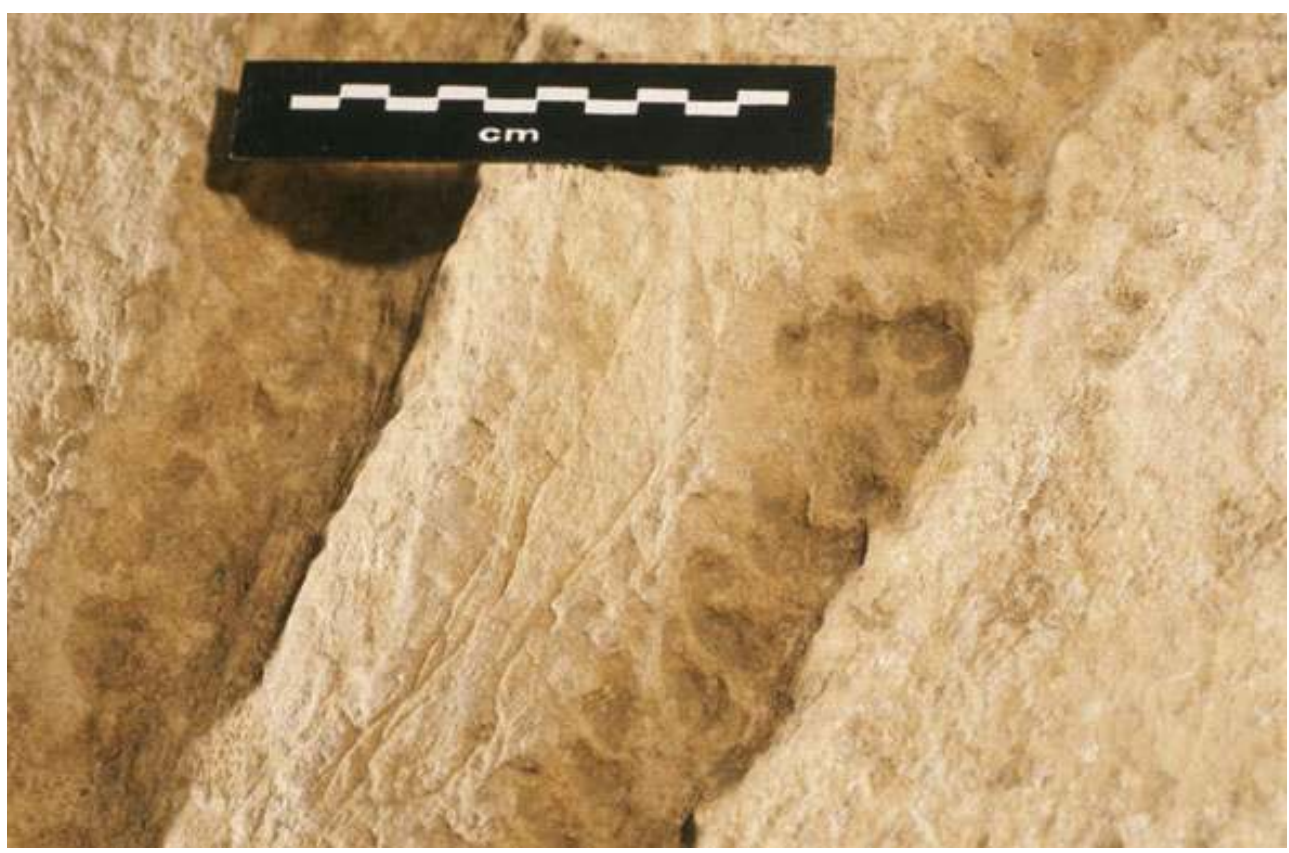

Panneau des femmes

Pinçon, G. (C) G. Pinçon et DRAC Poitou-Charentes, 1997

Plusieurs phases d'intervention des Magdaléniens s'observent sur la paroi : une première phase de gravures fines (fig. $\mathbf{n}^{\circ} \mathbf{6}$ ), sur la paroi naturelle juste dégagée des éléments instables puis martelée; une deuxième phase d'aménagement entraînant la chute de blocs gravés dans la couche d'occupation; une troisième phase de réaménagement, plus conséquente, avec creusement et aplanissement des surfaces pour préparer le plan des sculpteurs et une quatrième phase de retaille des sculptures entraînant la chute de fragments dans les couches.

\section{Le relevé analytique : un outil d'analyse précieux pour l'approche d'une chronologie pariétale}

Etape indispensable dans la démarche scientifique en art pariétal, le relevé aide à la lecture et aux nouvelles interprétations. Il s'appuie sur une analyse fine de la paroi et le décryptage des différents stigmates l'affectant. Il met en valeur le "polymorphisme technologique » des traits sculptés, gravés et peints ainsi que des accidents naturels, la préparation éventuelle du support par les artistes, les érosions, les dégradations anthropiques ou non des surfaces et permet alors d'appréhender les différentes étapes de réalisation des œuvres, leurs reprises et leur évolution au cours du temps ${ }^{11}$.

Le rôle du relevé d'art pariétal a évolué au cours du temps, de même que sa méthodologie. Le penchant actuel, d'autant plus renforcé par l'usage de l'informatique (photographies numériques, photogrammétrie, relevé 3D au laser, ...), est de tendre vers l'exhaustivité, mais un relevé scientifique demande toujours une sélection et une hiérarchisation des phénomènes à retranscrire. Il apparait comme un document subjectif puisqu'il impose des choix. Il est également un support visuel pour se faire comprendre de même que la carte. Il doit donc être clair et compréhensible par le plus grand nombre, pertinent et 
efficace. Il est à la fois support pour partager une connaissance, et moyen pour faire la démonstration d'un phénomène. Le relevé est donc différent du fac-similé ou de la « copie » qui visent une restitution parfaite.

Figure 7

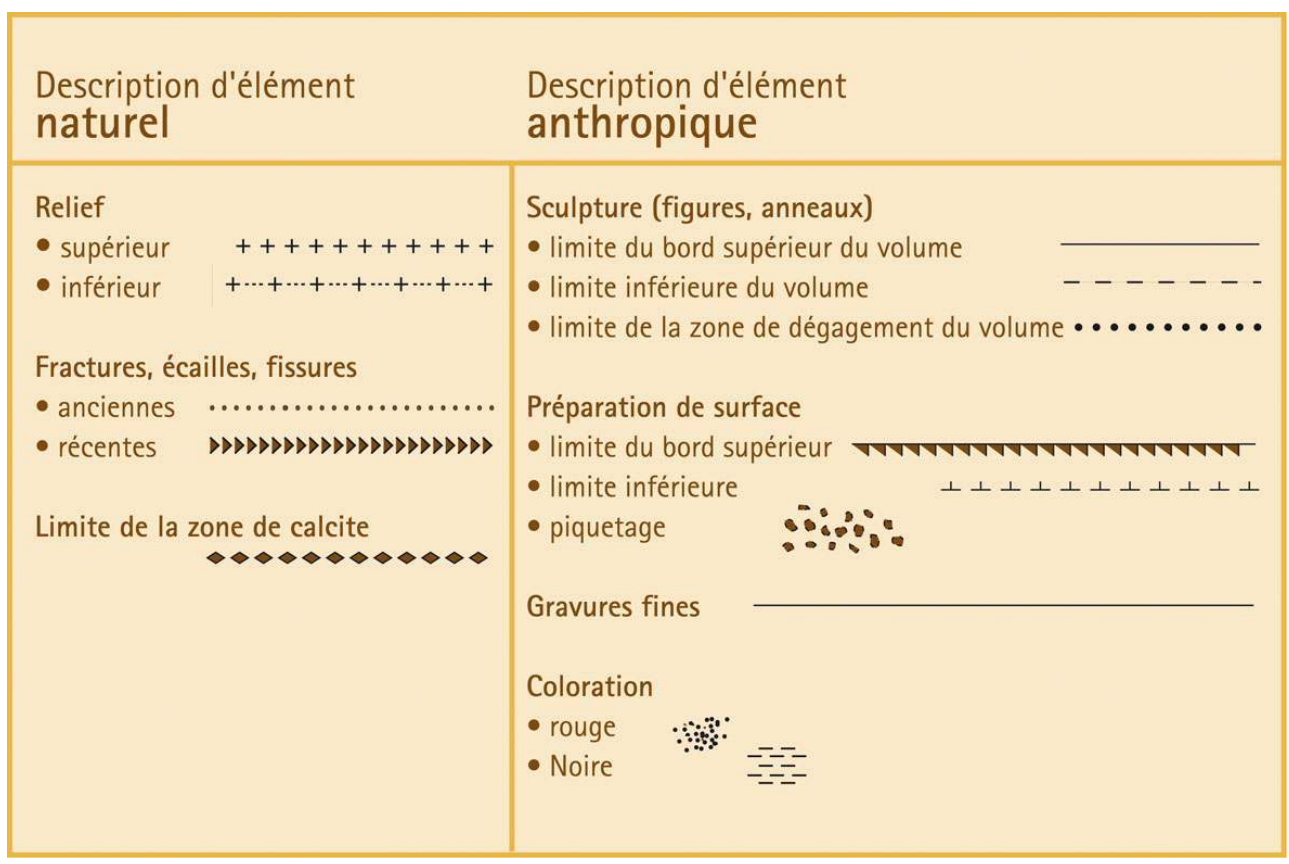

Charte graphique utilisée pour les relevés d'analyse graphique réalisés au Roc-aux-Sorciers (c) G. Pinçon graphiques et l'application d'une charte graphique (fig. $\mathbf{n}^{\circ} 7$ ). Le relevé graphique analytique s'appuie sur les figures fondamentales de la géométrie (lignes, points et surface), leur taille, leur teinte. Chaque détail est retranscrit selon la charte graphique définie suivant les conventions établies. Il s'agit de distinguer les éléments naturels (relief naturel, fissures ou fractures anciennes et récentes, calcite, cupules de gel, écailles, ...) des éléments anthropiques (gravures, reliefs anthropiques, traces de sculpture, traces de débitage et d'épannelage, éléments de reprise de sculpture, raclages, piquetages, vestiges d'une préparation de surface, colorations).

Le relevé graphique analytique est, par ailleurs, toujours associé à d'autres techniques de relevés complémentaires comme la photographie.

La numérisation ${ }^{12}$ du relevé graphique analytique obtenu fournit un support idéal pour réaliser un "relevé vecteur $~_{13}^{13}$ apte à aider à l'analyse et à l'interprétation des œuvres magdaléniennes (fig. $\mathbf{n}^{\circ} \mathbf{8}$ ). Tous les tracés du relevé graphique sont repris avec un logiciel de dessin, point par point, à l'aide d'une palette graphique, selon le code établi et sont organisés sur différentes feuilles superposées de calque. Chaque forme est donc redessinée de façon indépendante et peut être individualisée. Une bibliothèque des formes graphiques identifiées (ou unités graphiques) est alors créée (fig. $\mathbf{n}^{\circ}$ 9). Chaque représentation isolée dans la bibliothèque des formes peut alors être superposée, comparée, associée à une autre. Le rendu vecteur des figures permet d'agrandir les formes, de leur attribuer des codes couleurs distincts. La vectorisation du relevé analytique permet de simuler plusieurs interprétations à partir de différentes 
associations de traits. Elle permet aussi de préciser les éléments constituant une figuration et permet de formuler, si nécessaire, plusieurs hypothèses. Le "relevé vecteur » et la « bibliothèque de formes » qu'il permet de créer apportent ainsi, à travers leur exploitation, des éléments de réponse aux phénomènes de succession et d'évolution des formes qui éclairent sur la nature même de l'occupation de l'abri sous-roche sculpté.

Figure 8

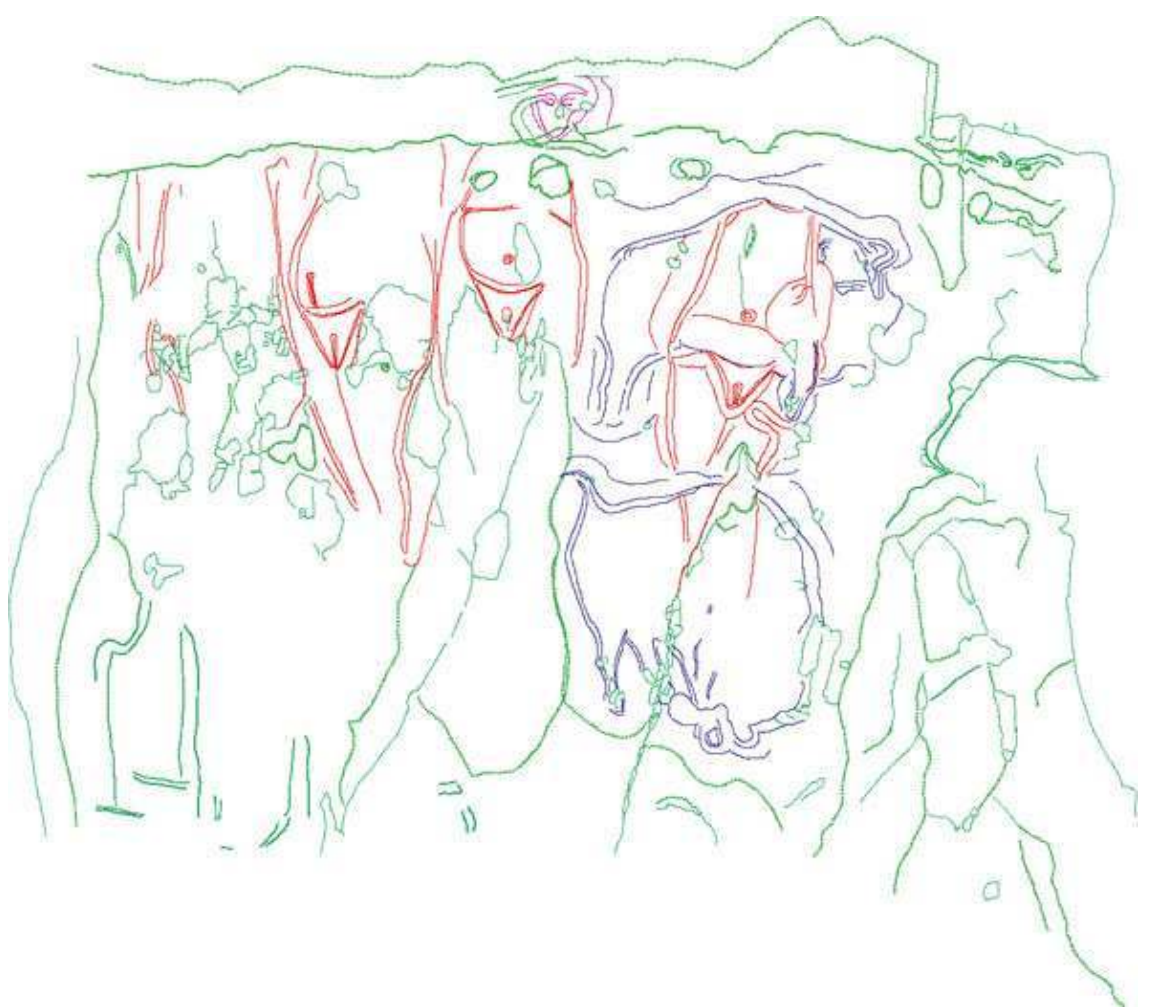

Exemple extrait du relevé analytique vecteur de la frise sculptée du Roc-aux-Sorciers

Relevé G. Pinçon, L. lakovleva et O. Fuentes @ G G. Pinçon 


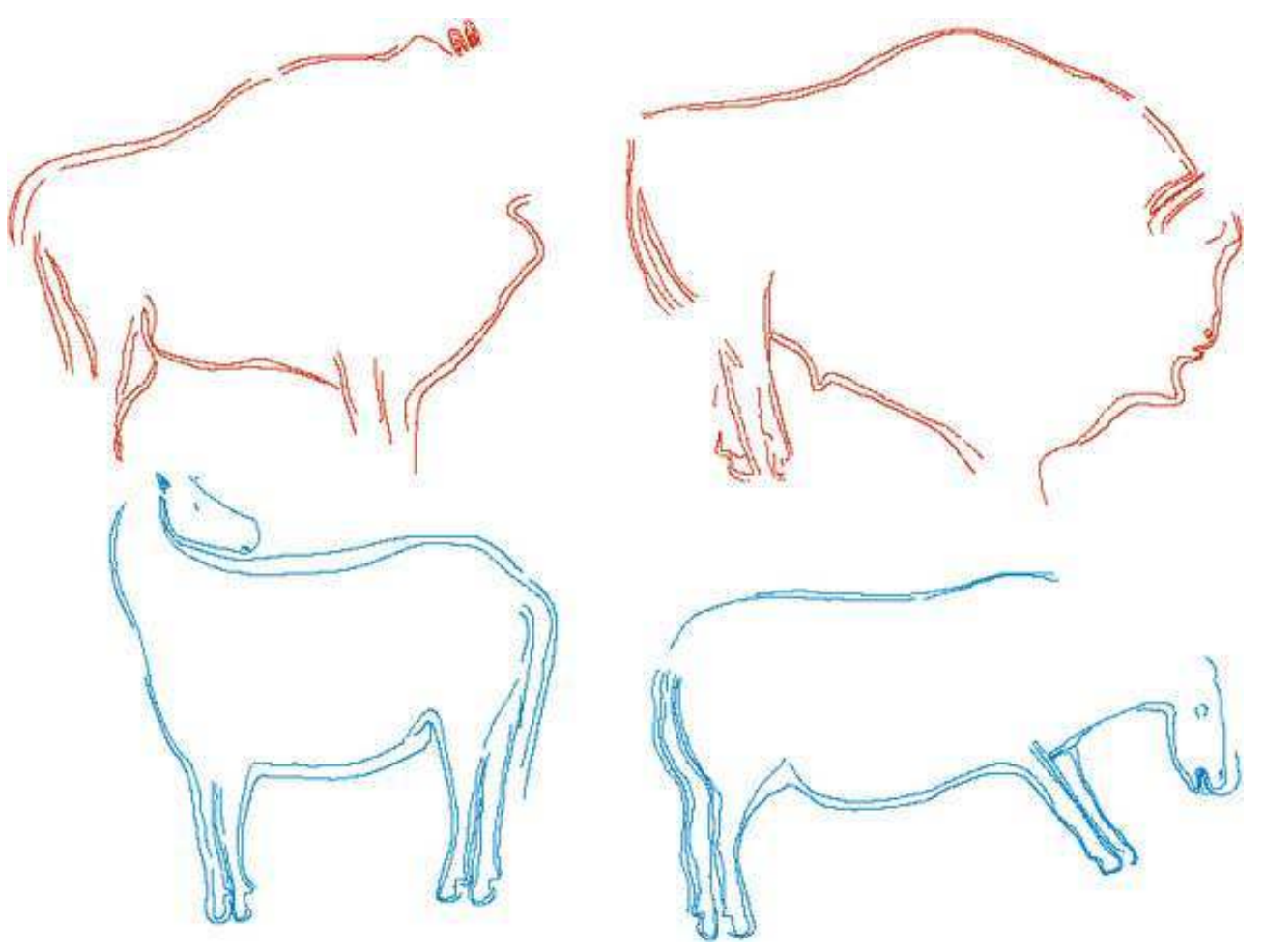

Exemple d'éléments de la bibliothèque de formes graphiques issues du relevé analytique de la frise sculptée du Roc-aux-Sorciers

Relevé G. Pinçon et O. Fuentes (C) G. Pinçon

\section{Lecture et interprétation des œuvres pariétales sculptées monumentales de la frise du Roc-aux- Sorciers}

L'outil d'analyse que constitue la bibliothèque des formes ainsi réalisée est utile dans le cadre des recherches menées actuellement sur le site et permet de proposer un certain nombre d'observations. Celles-ci viennent parfois valider des conclusions issues de l'étude graphique ou, parfois, apportent une lumière nouvelle sur certains aspects incompris jusque-là ou encore non interprétés. Le nombre de vestiges de sculpture présents sur la paroi est assez important, le relevé vecteur permet de proposer des hypothèses d'interprétation de certaines de ces formes.

La bibliothèque des formes réalisée à partir du relevé vectorisé permet également de mettre en évidence la redondance de certains caractères. Par la comparaison des éléments graphiques, on peut mettre en évidence l'homothétie de plusieurs figures. Cette analyse apporte des éléments sur le comportement des artistes vis à vis de telle ou telle figure.

L'art pariétal du Roc-aux-Sorciers est un art homogène, fruit de la création d'hommes ayant obéi à des règles très précises. Animaux et humains sont figurés avec un grand réalisme qui caractérise cet art pariétal. Tout en mettant en exergue les éléments propres à chacune des espèces figurées, l'artiste respecte les proportions des chevaux, bouquetins, 
bisons ou félins et apporte un traitement soigneux aux détails anatomiques. Les attitudes des animaux ont été choisis selon le espèces. A cela s'ajoutent les traits de stylisation particulière sur différentes figures comme la géométrisation de certains détails anatomiques marquant un style propre. Il en est ainsi du traitement en relief triangulaire des yeux des chevaux ou des sexes des femmes ou encore de la sculpture des museaux des bouquetins ou chevaux réalisés de façon à rendre une vue de face des naseaux. Ces éléments de stylisation et d'homogénéité entre les figures soulignent la similitude des images par espèce au sein de la frise sculptée de l'abri Bourdois et aident à l'interprétation des vestiges de sculptures.

\section{L'interprétation des figures partielles et l'analyse des phénomènes de retaille}

Figure 10

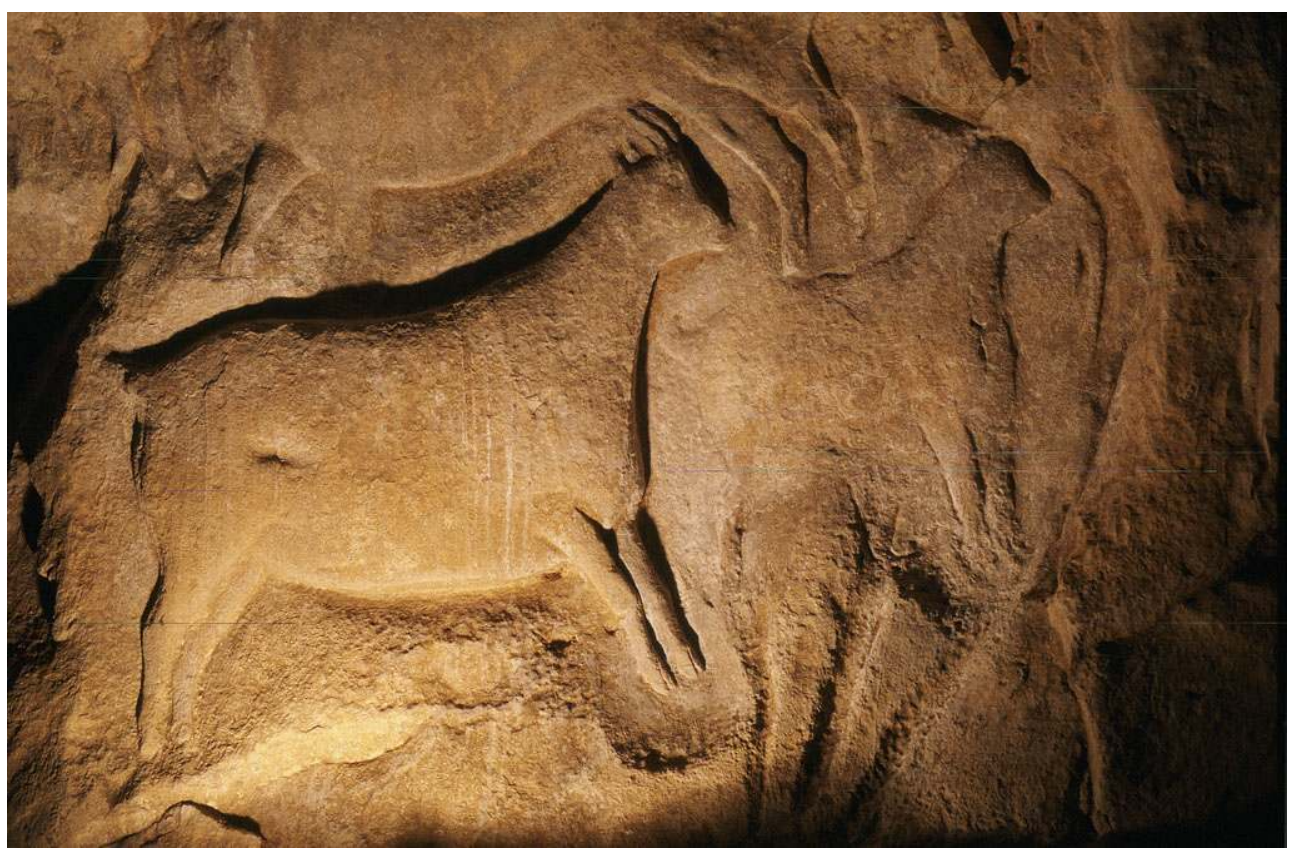

Etagne et jeune bouquetin sculptés sur la frise de l'abri Bourdois du Roc-aux-Sorciers

Pinçon, G. ( ) G. Pinçon et DRAC Poitou-Charentes, 2005

L'art pariétal paléolithique est souvent présenté de façon statique, à savoir une image à un instant précis. Il nous est livré aujourd'hui dans sa phase de réalisation finale, avec un état de conservation lié aux influences naturelles ou anthropiques subies au cours du temps.

Sur la frise sculptée du Roc-aux-Sorciers, juxtaposée à une étagne, un jeune bouquetin (Bo5) est figuré de profil droit en relief léger (fig. $\mathbf{n}^{\circ} \mathbf{1 0}$ ). On peut lire la tête avec le museau, le chanfrein, l'œil en amande et le départ de la corne. Juxtaposé, à droite, un autre sujet est vu de profil gauche (An1). Il est partiel. On peut suivre sa ligne de dos, sa croupe, sa fesse, sa queue longue en relief léger. Un éclairage rasant du panneau appuie la ligne de dos sur une plus longue distance, offrant ainsi la morphologie d'une ligne de dos de bison. Juste au-dessous du jeune bouquetin, les vestiges d'une femme sculptée (Vé5) sont également lisibles. Cette figure a été détruite en partie par la sculpture plus récente 
de bouquetin. En effet, la partie supérieure de la figure féminine a été récupérée lors de l'élaboration du jeune bouquetin. Le triangle pubien ainsi que les jambes ont été épargnés par cette retaille. Le positionnement des images de la bibliothèque des formes permettant de projeter des images plus complète de ces figures partielles (bison et femme) soulignent l'association parfaite de ces deux images lors de leur réalisation avant leur retaille pour laisser la place au jeune bouquetin (fig. $\left.\mathbf{n}^{\circ} \mathbf{1 1}\right)$.

Figure 11

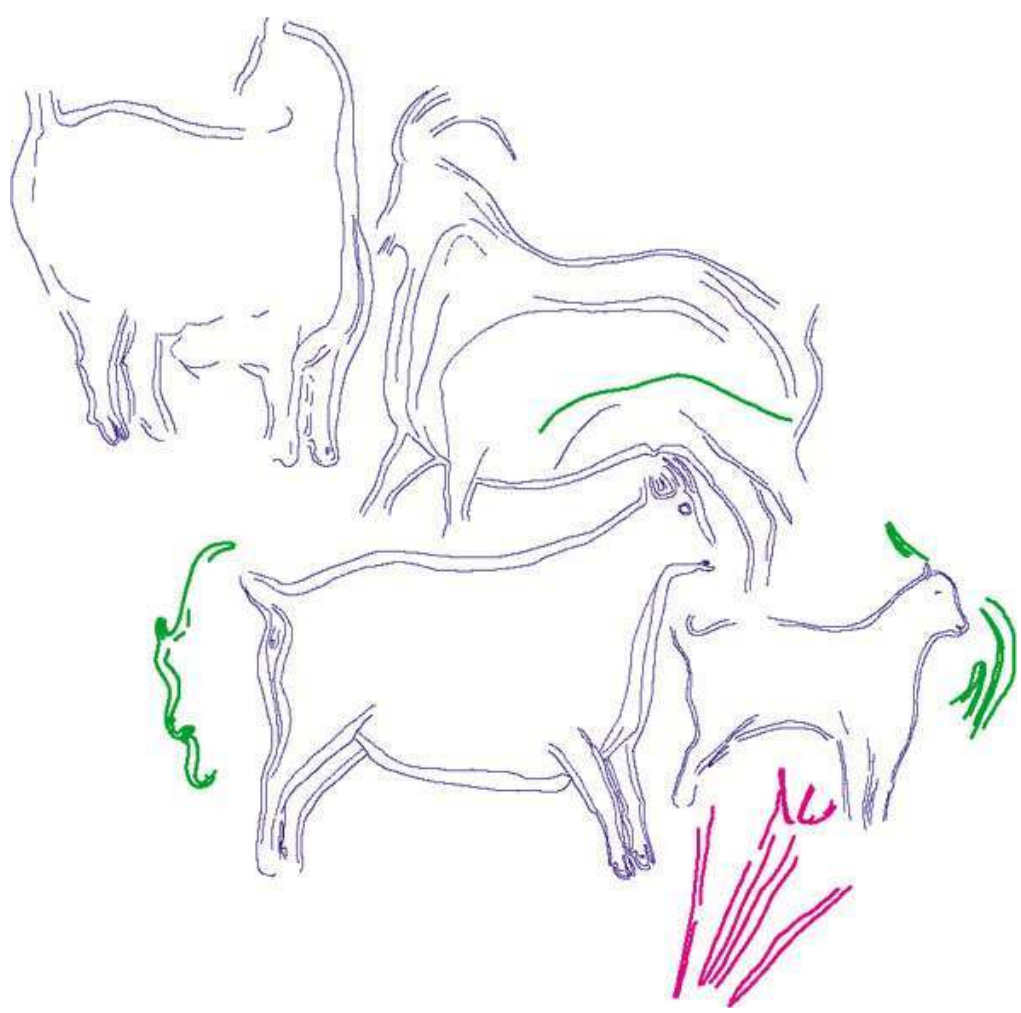

L'association femme (Vé5) / bison (An1) antérieure à la représentation de l'étagne (Bo6) et du jeune bouquetin (Bo5)

Relevé G. Pinçon, L. lakovleva et O. Fuentes @ C G. Pinçon

Deux incisions bien délimitées sur la surface concave forment le triangle pubien de la Vénus. Cette surface est polie. La première incision du triangle pubien (à gauche) est linéaire, à bout courbe, et la seconde est en forme de V. 
Figure 12

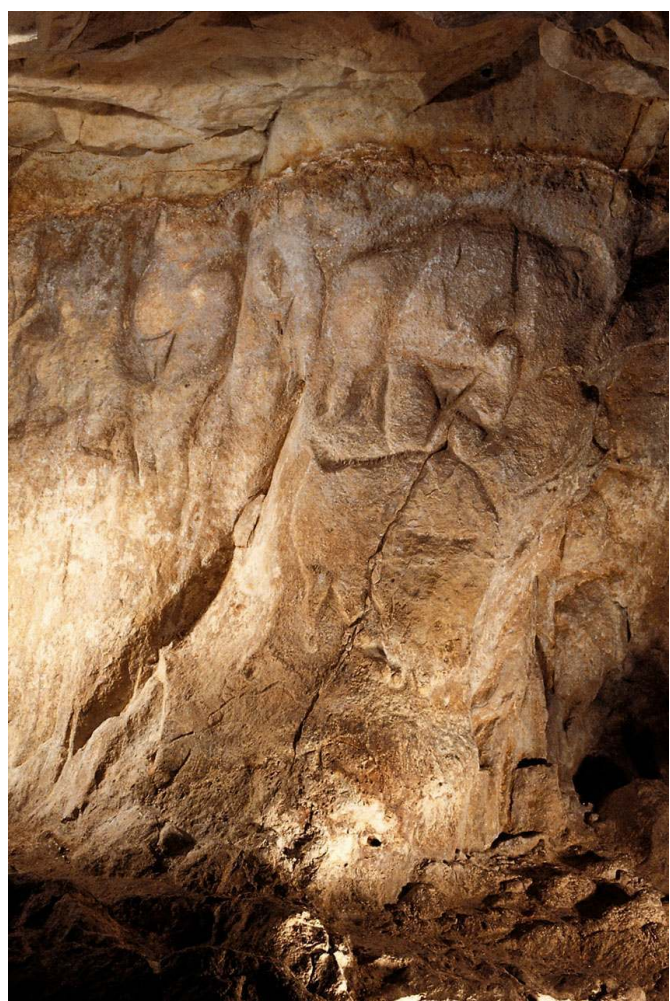

Femme / bison sculptés encore en place sur la frise de l'abri Bourdois du Roc-aux-Sorciers

Pinçon, G. (c) G. Pinçon et DRAC Poitou-Charentes, 1997

L'incision linéaire est en $U$, plus fine que l'incision en $V$ qui est large et dissymétrique, dégageant ainsi plus de volume pour la partie gauche du sexe de la Vénus. Or le recoupement avec les figures complètes se calant parfaitement sur les deux vestiges de sculpture sans déformation morphologique ni morphométrique, marque l'association forte entre figure féminine et bison, le sabot du bison s'impliquant dans le sexe de la femme. Ce thème femme-bison, fréquent dans l'art pariétal, est cependant toujours présent de façon explicite dans la frise encore in situ (fig. $\mathbf{n}^{\circ}{ }^{12}$ ). L'intégration de nouvelles figures de bouquetin n'a pas complètement effacé les images et thèmes plus anciens. Les interventions successives des sculpteurs introduisant de nouvelles figures se font dans la continuité.

\section{L'intervention des Magdaléniens sur leurs propres œuvres}


Figure 13

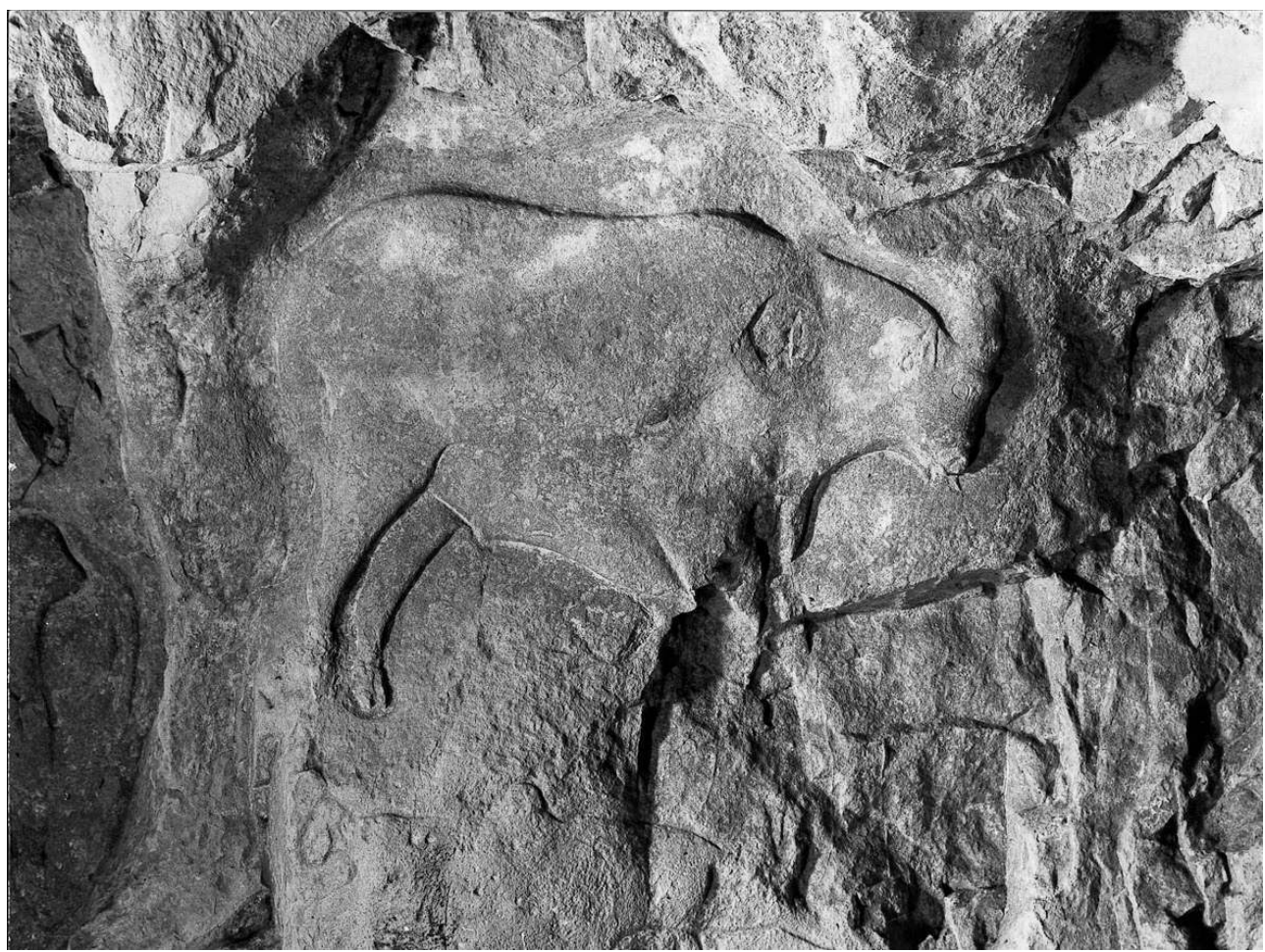

Bouquetin mâle (Bo7) issu d'anciennes figurations de bison et de cheval. de Saint-Mathurin, S

(c) Musée d'archéologie nationale, fonds Saint-Mathurin

Plus à droite sur la frise sculptée de l'abri Bourdois, un bouquetin mâle tirant la langue (Bo6), vu de profil droit, est sculpté en haut relief (fig. $\mathbf{n}^{\circ}$ 13). Seules ses pattes antérieures sont fracturées. Sa tête est bien dégagée et porte les caractéristiques d'une tête de bouquetin mâle. L'œil, l'oreille, le naseau sont détaillés. Les cornes courbes, en arrière, sont présentes par leur volume. 


\section{Figure 14}

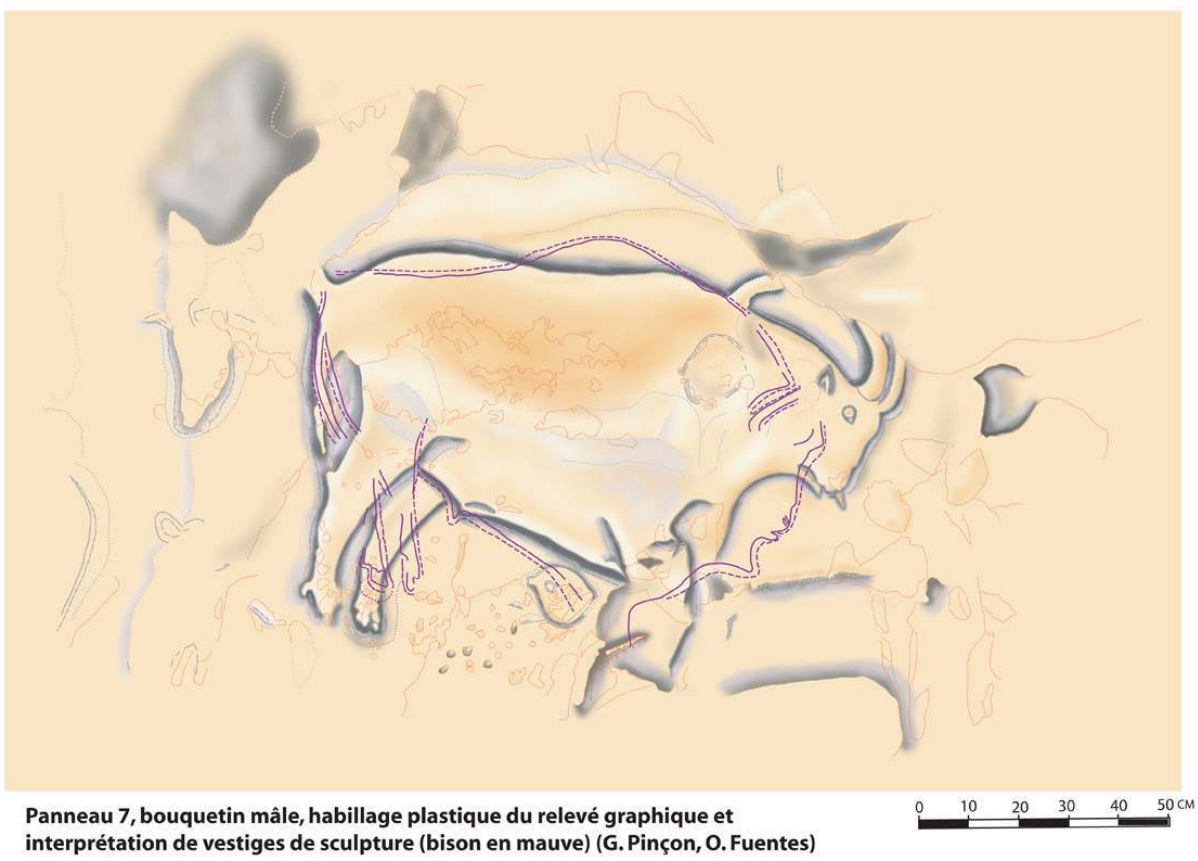

Relevé du bouquetin mâle avec proposition d'interprétation à l'aide de la bibliothèque de formes vecteur des négatifs des vestiges sculptés : forme d'une image ancienne de bison possible

Relevé G. Pinçon, L. lakovleva et O. Fuentes @ G. Pinçon

\section{Figure 15}

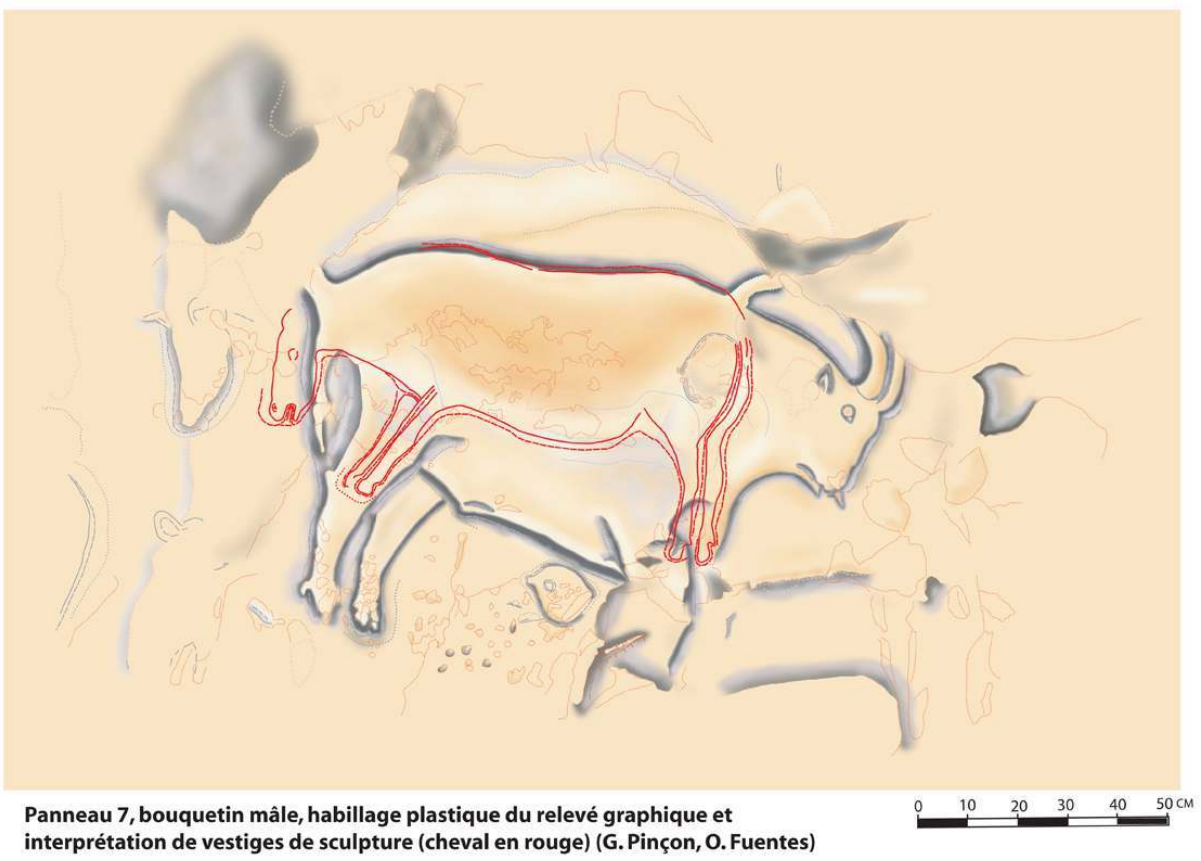

Relevé du bouquetin mâle avec proposition d'interprétation à l'aide de la bibliothèque de formes vecteur des négatifs des vestiges sculptés : forme d'une image ancienne de cheval possible

Relevé G. Pinçon, L. lakovleva et O. Fuentes ( $)$ G. Pinçon 
dant, la ligne de dos marque une convexité forte au niveau du garrot qui apparaît anguleux, trop anguleux pour un sujet bouquetin. Son corps au niveau du garrot est également trop large pour un bouquetin. La zone de dégagement de son dos est particulièrement large, réalisée à l'aide de raclages. Elle marque une zone très convexe juste au-dessus du garrot et évoque une bosse de bison vue de profil droit (fig. $\mathbf{n}^{\circ}$ 14). Il s'agit probablement d'un bison retaillé comme l'avait déjà suggéré S. de Saint-Mathurin.

Des négatifs de sculpture sont également visibles à l'intérieur même du bouquetin. Par l'observation de la paroi et les jeux d'éclairage, une forme de cheval se dégage. La ligne de dos du bouquetin sert alors également comme ligne de dos d'un possible cheval ayant existé avant le bouquetin. La forme du cheval rappelle la posture de l'équidé sculpté inclinant la tête, situé plus en amont de la frise (fig. $\left.\mathbf{n}^{\circ} \mathbf{1 5}\right)$.

33 A partir de la bibliothèque, il est possible de faire des superpositions permettant d'illustrer ces interprétations. Le rendu paraît corroborer les propositions de lecture. Le cheval cadre parfaitement avec les points d'accroche de sculpture. De même la ligne évoquant la courbe cervico-dorsale d'un bison aide à placer l'image de bison transformée en bouquetin.

'histoire de l'évolution artistique de cette figure de bouquetin actuellement en place sur la frise peut être ainsi évoquée: cette image de bouquetin a été retaillée dans une ancienne image de bison et peut-être également de cheval.

Ces nouvelles interprétations viennent compléter la vue d'ensemble que l'on a de la frise, notamment, la variété des figures représentées au cours d'un millénaire d'occupation magdalénienne. La frise a connu une évolution des thèmes sculptés. Certaines figurations ont été effacées (bison, cheval, femme), d'autres retaillées (bouquetin). Certaines représentations ont été respectées de tout temps (bison, femme, cheval). L'image que l'on a de la frise sculptée encore in situ dans l'abri Bourdois correspond à une image du dernier état artistique. Les étapes antérieures de création peuvent être ainsi appréhendées et on peut donc retrouver l'état de la frise à des époques antérieures à celle retrouvée.

Cependant, la fragilité de notre analyse repose sur les outils utilisés, à savoir des images issues du relevé analytique, réalisé en deux dimensions. La base de données 3D de la frise sculptée nous permet aujourd'hui de confronter ces observations en exploitant également la troisième dimension, incontournable dans l'analyse de ces œuvres sculptées. L'enregistrement laser 3D apparaît comme particulièrement adapté aux œuvres sculptées. Il parvient ainsi à retranscrire la troisième dimension des œuvres, sans distorsion dans les dimensions, dans les proportions, ni dans leur position relative réelle. Son échelle de résolution étendue lui permet de saisir avec grande précision les différentes nuances de tracé depuis la gravure fine jusqu'au haut-relief. L'enregistrement laser 3D offre un rendu fidèle et pertinent des figures, des formes générales jusqu'aux détails les plus ténus. Les technologies 3D viennent consolider nos interprétations de figures partielles (fig. $\mathbf{n}^{\circ} \mathbf{1 6}$ ). Le modèle 3D permet de projeter non plus seulement des formes comme celles issues du relevé vectorisé 2D, mais surtout des volumes. La 3D vient conforter nos hypothèses sur les reprises de sculptures en proposant des volumes plausibles aux figures aujourd'hui en partie disparues. Ces modélisations visent à recréer les différents états de la paroi au cours du Magdalénien, les diverses étapes dans l'élaboration de cette frise, avec leurs évolutions techniques et thématiques ${ }^{14}$. 


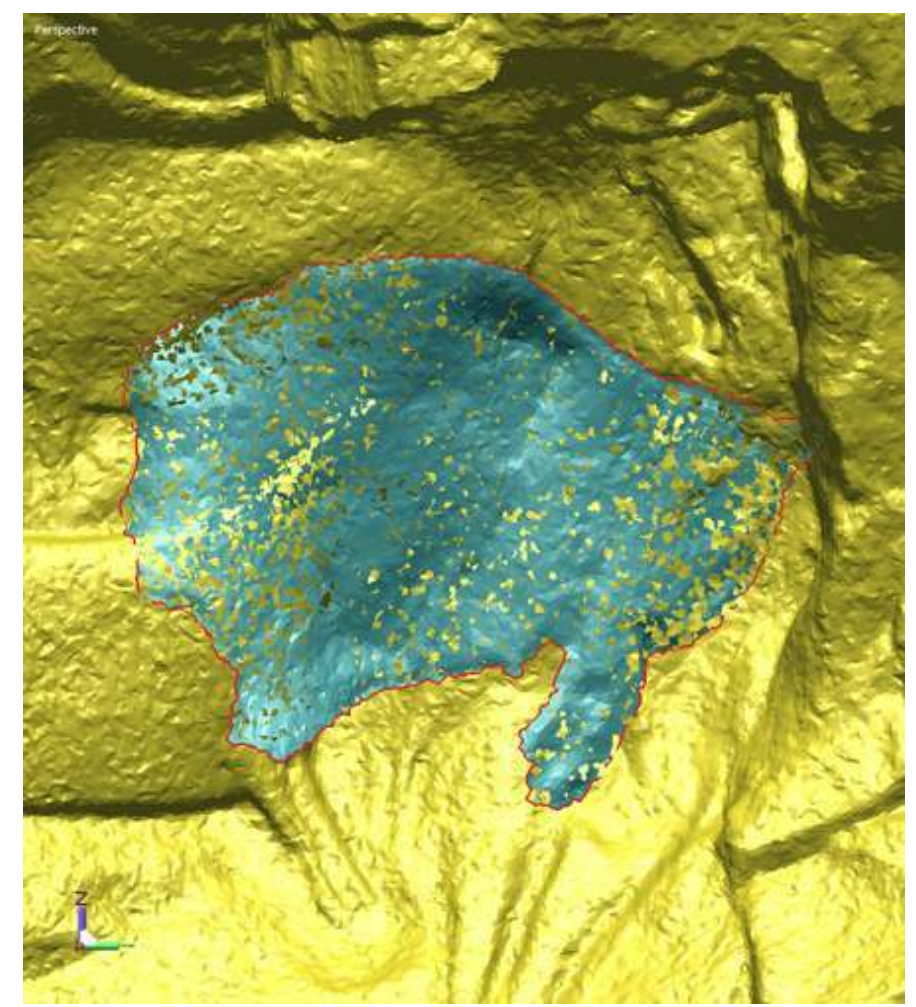

Exploitation de la bibliothèque des formes 3D pour aider à l'interprétation du vestige de sculpture de bison effacé au profit de la sculpture du jeune bouquetin

(c) G. Pinçon, DRAC Poitou-Charentes, Art graphique et patrimoine

\section{Conclusion}

Ainsi, un bouquetin mâle est issu d'anciennes figurations de bison et de cheval. Une association femme-bison est antérieure à la représentation d'un jeune bouquetin. Nous constatons donc au Roc-aux-Sorciers, une appropriation continue de la paroi au cours de l'occupation par les magdaléniens. Certaines images anciennes sont retaillées pour exprimer les nouvelles thématiques mais, en même temps, les anciennes thématiques sont conservées intégralement ou partiellement malgré tout sur la frise. Les retailles ne sont pas le signe d'un manque de respect des traditions. Le résultat est une frise offrant le reflet d'images de différentes périodes, entre tradition et innovation, marquant en quelque sorte leur intemporalité.

Mais s'agit-il de différents sculpteurs magdaléniens? La question de la durée du temps de création des œuvres sur la paroi se pose. Il nous est encore difficile, dans l'état actuel de notre connaissance, de définir l'espace de temps qui sépare chaque intervention sur la paroi, entre les magdaléniens qui s'installent les premiers sur ce site et l'événement naturel qui entraîne l'effondrement brutal juste après l'occupation de ces magdaléniens, un millénaire plus tard.

Plusieurs phases d'intervention s'observent ainsi sur les parois : une première phase de gravures fines ; une deuxième phase d'aménagement entraînant la chute de blocs gravés dans la couche d'occupation; une troisième phase de réaménagement, plus conséquente, 
avec creusement et aplanissement des surfaces pour préparer le plan des sculpteurs où les thèmes femme / bison / cheval dominent et une quatrième phase de retaille des sculptures avec l'introduction du thème nouveau du bouquetin, entraînant la chute de fragments dans les couches. Les sculptures qui portent des traces de retaille soulignent une appropriation continue de la paroi par les Magdaléniens et le respect de règles de représentation (morphométrie, morphologie, détails anatomiques stylisés, etc.), partagées par le groupe lors de chaque intervention sur la paroi.

Récemment, des tentatives de comparaisons homothétiques ont été faites entre les frises sculptées magdaléniennes du Roc-aux-Sorciers et de la Chaire-à-Calvin à Mouthiers-surBoême (Charente, France). De multiples parallèles sont apparus entre ces deux ensembles sculptés de la même période. Les comparaisons homothétiques, en deux et trois dimensions, se révèlent particulièrement précieuses pour l'analyse de l'iconographie pariétale d'un site mais aussi dans l'établissement de territoires artistiques à travers des comparaisons inter-sites. Au-delà de l'appartenance à une tradition artistique et symbolique commune de ces deux sites, une telle conformité entre deux œuvres distantes de plus de $150 \mathrm{~km}$ soulève d'autres questions: ont-elles été exécutées par le même artiste? La transmission et la reproduction de codes techniques et graphiques à l'identique est-elle possible dans le temps et / ou l'espace?

\section{NOTES}

1. Breuil, Henri. Quatre cents siècles d'art pariétal. Centre d'Etudes et Docum. Préhist., Montignac, 1952.

2. Leroi-Gourhan, André. Préhistoire de l'art occidental. Paris : $1^{\mathrm{er}}$ éd. Mazenod, 1965.

3. Lorblanchet, Michel. De l'art des grottes à l'art de plein air au Paléolithique. L'Art paléolithique à l'air libre, le paysage modifié par l'image, 7-9 octobre 1999, UMR 5590 du CNRS - Tautavel-Campôme, 2002, Tautavel, GAEP \& GEOPRE, p. 97-112.

4. Voir dans la base Mérimée : notice PA00105323.

5. Voir le site : http://www.roc-aux-sorciers.com/la_dycouverte.php

6. Voir le site : http://www.roc-aux-sorciers.com/la_dycouverte.php

7. Voir le site : http://www.roc-aux-sorciers.com/la_dycouverte.php

8. Voir le site : http://www.roc-aux-sorciers.com/la_dycouverte.php

9. Pinçon, Geneviève. Rapport d'activité sur le site du Roc-aux-Sorciers à Angles-surl'Anglin (Vienne), 2007.

10. Pinçon, Geneviève, Iakovelva Ludmila. Angles-sur-l'Anglin. La frise sculptée du Roc-auxSorciers. Paris : Ed. R.M.N. et C.T.H.S., 1997. Voir aussi le site : www.roc-aux-sorciers.com

11. Tymula, S. L'art solutréen du Roc de Sers (Charente). Paris : DAF, Édition de la Maison des Sciences de l'Homme, 2002. p. 134.

12. La numérisation consiste dans ce cas en un balayage ligne par ligne, chaque ligne étant composée de points élémentaires jointifs (pixels).

13. Chaque objet du relevé est décrit par des points successifs composant son pourtour (mode vecteur).

14. Ce travail est actuellement en cours et fera l'objet d'une autre publication. 


\section{RÉSUMÉS}

L'abri sous-roche magdalénien du Roc-aux-Sorciers (Angles-sur-l'Anglin, Vienne) joue un rôle essentiel et novateur pour l'enrichissement de notre connaissance sur l'art pariétal sculpté en raison de l'impact de cette technique sur les œuvres monumentales qu'il livre. Ayant constaté à plusieurs reprises des phénomènes de retaille sur la paroi, ainsi que la présence de vestiges de sculpture volontairement effacés, nous abordons la chronologie des œuvres pariétales en rappelant le contexte archéologique de ces œuvres et en analysant sur quelques exemples l'évolution des thèmes artistiques, les choix iconographiques perceptibles au cours de l'occupation de ce site.

The magdalenian rock-shelter of Le Roc-aux-Sorciers (Angles-sur-l'Anglin, Vienne) is playing an essential and innovative role in the enrichment of our knowledge of sculpted parietal art, given the impact of this technique on the monumental work it produces. Having noted several examples of recarving on the wall, as well as the presence of traces of sculpture that have been deliberately erased, we tackle the chronology of the parietal works, emphasising their archaeological context and analysing through several examples the evolution of the artistic themes, the iconographic choices which are perceptible during the site's occupation. (traduction : Paul Bahn)

\section{INDEX}

Mots-clés : Magdalénien, Roc-aux-Sorciers, Angles-sur-l'Anglin, art pariétal, sculpture, chronologie, Paléolithique, Lascaux, grotte Chauvet, abri sous roche, datation relative, relevé analytique, retaille, vestiges, paroi, système symbolique, habitats, gisement, site, cavité karstique, grotte, cave Taillebourg, abri Bourdois, Magdalénien moyen, bisons, chevaux, femmes, félins, bouquetins, hauts-reliefs, bas-reliefs, gravure, peinture, anneaux, art naturaliste monumental, éléments de style, gravures fines, aménagements, numérisation, relevé vecteur, bibliothèque des formes graphiques, abri sous-roche sculpté, influences naturelles, influences anthropiques, Vénus, base de données 3D, laser 3D, modèle 3D, relevé vectorisé 2D, blocs gravés

\section{AUTEUR}

\section{GENEVIÈVE PINÇON}

Chercheur. Sous-direction de l'archéologie, de l'ethnologie, de l'inventaire et du système d'information, direction de l'architecture et du patrimoine. UMR 7041 Arscan. genevieve.pincon@culture.gouv.fr 